\title{
A Precautionary Technique for the Accidental Ingestion of Preformed Zirconia Crowns in Pediatric Dentistry
}

Shital Kiran ${ }^{1}$, Hetal Majeethia², Mehul Jani ${ }^{3}$

\begin{abstract}
Accidental ingestion of the preformed zirconia crown may occur if the dental treatment is carried out without using rubber dam in the children. Mishaps are rare in adults, but in pediatric patients foreign body ingestion is a global problem that is potentially harmful to the patients, which may lead to life-threatening condition and it also causes anxiety to the clinician. This paper elaborates a simple technique to tag zirconia crown with the suture thread using resin-modified glass ionomer luting cement to prevent accidental ingestion in the routine pediatric dental practice as a reserved technique for patients who are unable to accept rubber dam.

Keywords: Emergency, Ingestion, Preventive technique, Zirconia crowns.

Journal of South Asian Association of Pediatric Dentistry (2021): 10.5005/jp-journals-10077-3056
\end{abstract}

\section{INTRODUCTION}

Occurrences of foreign body (FB) ingestion are very high in pediatric patients and also in the special needs children due to behavior problems. Frequently aspirated substances were peanuts followed by plastics, metals, and toys in children. ${ }^{1}$ Pediatric dental patients exhibit sudden jerky and unpredictable movements that may lead to undesirable accidents and the same can be noticed even in adults. ${ }^{2}$

Frequently used dental instruments used in the routine clinical procedures may be swallowed accidentally. The teeth, endodontic instruments, crowns, rubber dam clamp, restorative and impression materials, gauze packs, and fixed prosthodontic appliances are prone to ingest followed by orthodontic appliances. ${ }^{3}$ The occurrences of aspiration and ingestion during the dental procedure have been documented by numerous articles and reviews as early as in 1971, Grossman stated that $87 \%$ of FBs entered the alimentary tract, whereas $13 \%$ aspirated into the respiratory tract under the age of 20 years. ${ }^{4}$

Notably, $80-90 \%$ of FBs in the gastrointestinal tract are passed freely without any complications, $10-20 \%$ are withdrawn endoscopically, and $1 \%$ requires surgery. In 2000, the American Association of Poison Control Centers documented 116,000 FB ingestions and it was found that $75 \%$ FB was reported in children aged $\leq 5$ years. ${ }^{5}$ Kurkciyan et al. ${ }^{6}$ stated that dental procedures involving single-tooth cast or prefabricated restorations involving cementation had a higher probability of aspiration and to prevent aspiration of these object tagging of objects with dental floss or suture material had been suggested.

A dental crown is difficult when it comes to anchoring or secured tagging. Anchoring the crown with floss is even more necessary when it is being delivered to special needs children, sedated children, physically disable individuals, or those being treated under general anesthesia as they have restricted protective laryngeal reflexes. For the stainless steel crown (SSC), Katge and Pammi have suggested a tagging technique with by anchoring the orthodontic band material on the middle third of crown followed by tying dental floss to the welded band. ${ }^{7}$ Kher and Rao have reported the use of medical grade tape on the occlusal surface of the stainless crown for its securement before trying out in the mouth for size
1,2Department of Pedodontics and Preventive Dentistry, College of Dental Sciences, Amargarh, Bhavnagar, Gujarat, India

${ }^{3}$ Private Practitioner in Oral and Maxillofacial Surgery, Bhavnagar, Gujarat, India

Corresponding Author: Shital Kiran, Department of Pedodontics and Preventive Dentistry, College of Dental Sciences, Amargarh, Bhavnagar, Gujarat, India, Phone: +91 7016310949, e-mail: drdpsk@gmail.com

How to cite this article: Kiran S, Majeethia H, Jani M. A Precautionary Technique for the Accidental Ingestion of Preformed Zirconia Crowns in Pediatric Dentistry. J South Asian Assoc Pediatr Dent 2021;4(1): 58-61.

Source of support: Nil

Conflict of interest: None

selection. ${ }^{8}$ The zirconia crowns, which fit passively and also due to its preparation involving blood and excessive salivation, make crown even more slippery which increases the chances for the accidental ingestion of the crown.

The purpose of this paper is to showcase an innovative technique involving routinely used dental material to secure preformed zirconia crown, which in turn can prevent accidental ingestion in pediatric dental practice.

\section{Case Description}

A 5-year-old girl reported to the pediatric dentistry clinic with the chief complaint of pain in her right lower back region since 15 days. Family and medical history were found to be noncontributory. Extraoral examination was not significant and intraoral examination showed dental age-appropriated dentition and dental caries with respect to $74,75,84$, and 85 had a deep carious lesion with tender to percussion (Fig. 1). The patient exhibited Frankel's behavioral rating scale II. Intraoral periapical radiograph revealed dental caries involving pulp in relation to 84 and 85 (Fig. 2).

After obtaining written consent from the parents for the treatment procedures. Pulpectomy was planned for the 74, 75, 84 , and 85 followed by a crown. Right inferior alveolar nerve block was administered to anesthetize both the primary first and second molars using $2 \%$ lignocaine with 1:80,000 adrenaline 


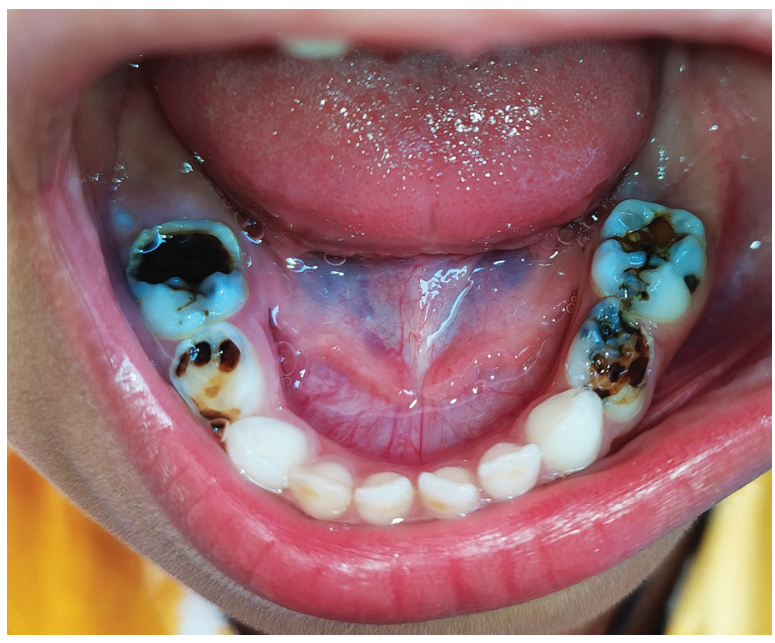

Fig. 1: Preoperative lower arch dental caries of 74, 75, 84 and 85

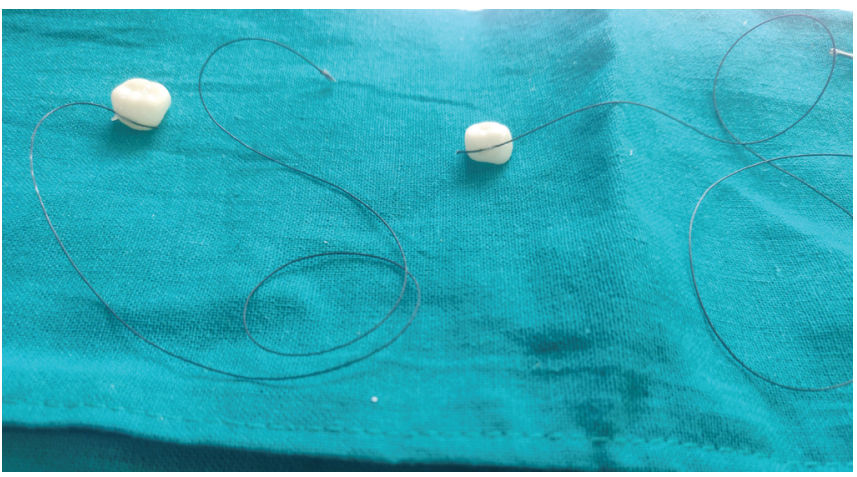

Fig. 3: Preselected zirconia crown with suture luted with resin modified glass ionomer luting cement

(Lignox, Indoco Remedies Ltd., Mumbai, India). Access opening was performed for both the molars followed by removal of coronal tissue and all canals were located using appropriate size K-file. After working length determination, biomechanical preparation was performed with Kedo-S square Ni-Ti rotary instruments and obturated with calcium hydroxide with iodoform (Tehno Dent lodotin paste) followed by coronal seal with glass ionomer cement (GC Fuji II, Tokyo, Japan). As the mother and father were highly concerned with the esthetic appearance of the restoration, it was decided to restore with a pediatric zirconia crown.

The selection of the appropriate zirconia crown size was performed on the cast model of patient prior to the second visit scheduled for tooth preparation by considering the mesiodistal dimension and also the crown size based on the original size of the tooth. The first molar mesiodistal dimension was $8.0 \mathrm{~mm}$ and for second molar it was $9.5 \mathrm{~mm}$. Crown size of D-LR-4 and E-LR-4 for the first and second molars was selected (Signature crowns, 3M Preformed Zirconia Crowns).

Local anesthesia was given prior to the tooth preparation. Using the marginal ridge of the adjacent teeth as a reference point, $1-1.5 \mathrm{~mm}$ of occlusal reduction was performed with flame-shaped diamond bur. Approximately $0.5-0.75 \mathrm{~mm}$ of the tooth structure was cut in the interproximal area with round end taper diamond bur and $0.5-0.75 \mathrm{~mm}$ buccal lingual reduction was performed using a round end taper diamond bur keeping all the natural contour of the original tooth. The reduction was initiated at the gingival margin

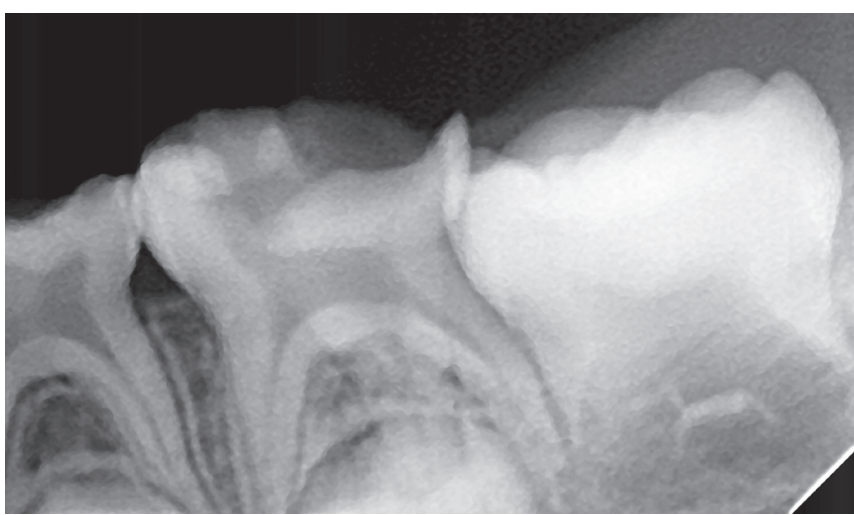

Fig. 2: Preoperative radiograph with dental caries involving 84 and 85

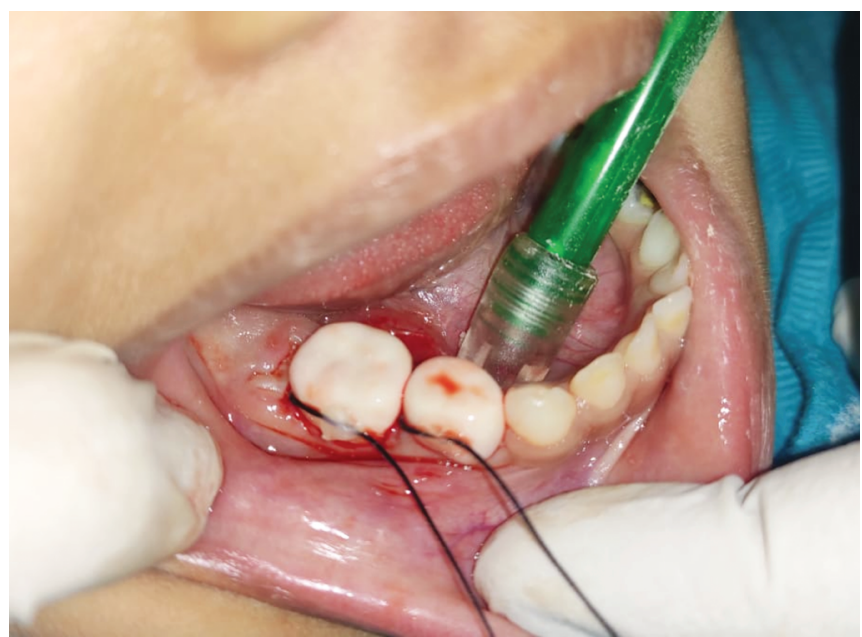

Fig. 4: Cementation of zirconia with resin modified glass ionomer luting cement

and was carried on to the occlusal plane with a rounded transition. The preparation was extended $0.5-0.75 \mathrm{~mm}$ into subgingivally extending up to the cementoenamel junction and finally the undercuts and sharp angles were removed and smoothed in order to allow for a completely passive fit of the crown. Try-in of the crown was checked for the passiveness of crown fit. In our case, the novel method for securing zirconia crown was followed using suture thread as an anchorage to prevent accidental ingestion.

After selecting zirconia crown for mandibular first and second molars as per the premeasured size from the cast, a non-absorbable black braided silk suture thread (lifeline) measuring 12" in length was placed on the middle aspect of the buccal surface of D-LR-4 and E-LR-4 and luted with resin-modified glass ionomer (RMGI) luting cement (GCFuji CEM 2 second-generation) 1 hour prior to the clinical procedure in order to save the time during clinical procedure (Fig. 3). Hemostasis of the gingiva was obtained by the gingival retraction cord (Ultrapak) with hemostatic (Medicept) agent, which was gently packed into the gingival sulcus around the specific tooth which was removed after adequate hemostasis and gingival displacement. Zirconia crowns were cemented with RMGI luting cement (GCFuji CEM 2 second-generation) prior to the removal of anchor (Fig. 4). After completion of the clinical procedure, the anchor was removed by just pulling the thread from the surface of crown (Fig. 5). Graphical representation explaining the technique used (Fig. 6). 


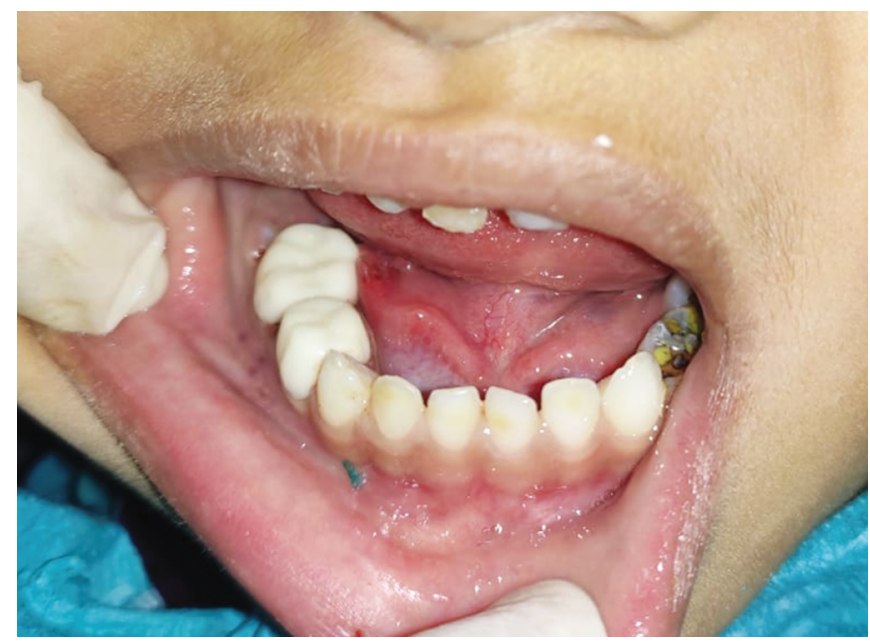

Fig. 5: Postoperative cementation of the crown

\section{Discussion}

Emergency is the word which raises the eyebrows for every pediatric dentist. Accidents may occur in the dental operatory in the form of FB ingestion, especially in pediatric patients as a result of the wobbliness of children. Unpredictable movements and sudden jerky movement are very common in children are the main cause for the undesirable incidences. The commonly used dental instruments that can cause ingestion during clinical procedures in pediatric dentistry are rubber dam clamp due to its springy property, SSCs because of the slippery surfaces, and endodontic instruments due to smooth short handle.

Zirconia crowns are a positive option due to their appealing esthetics, fast application, and superior hardness compared with other crown materials. They may be especially useful when esthetics are a concern or in case of uncooperative child. ${ }^{9}$ Ingestion or aspiration of semi-permanent restorations usually occur during the trial evaluation or during cementation. In pediatric dentistry, working with a rubber dam is not easy as in adult as sometimes it can change the behavior of the child. Although zirconia crowns are passive fitted due to its preparation, the blood and excessive salivation make crown more slippery and play a major role and there are more chances of slipping and accidental ingestion of the crown.

Various methods for the prevention of crown ingestion have been described in the literature, which is for the stainless crowns and as per our knowledge no case is being reported on the zirconia crowns. Since the zirconia crowns cannot be etched or bond, we used silk suture thread which had adequate adhesion of the zirconia crown with the RMGl luting cement. The black braided silk suture thread was placed on the middle aspect of the buccal surface of the mandibular posteriors in our case, similarly for the labial surface of mandibular anterior, palatal surface for the maxillary posteriors, and facial surface for the maxillary anterior crown may be used to attach the silk suture thread. The try-in of the crown which is used more often than the fitting crown carries more risk for swallowing, therefore employing our method may prevent the crown ingestion.

The RMGI cement resin bonds to the tooth structure, thereby preventing dissolution of the glass ionomer even from the inner part of the crown and also bonds to the receptors of the zirconia crowns. ${ }^{10}$ After cementation of the zirconia crowns with RMGI luting cement, the suture thread was removed by just pulling it. The risk of suture thread to stick on to the bur was always present,
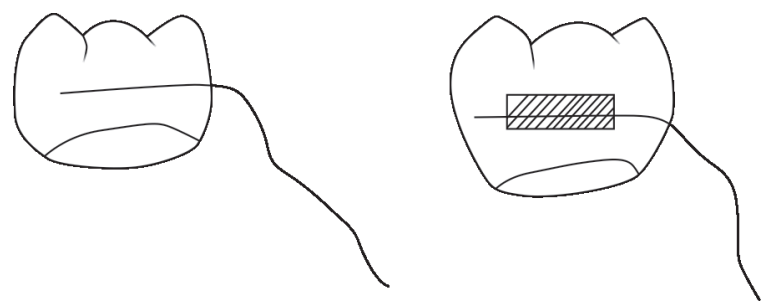

Fig. 6: Graphical representation of the technique

therefore after removal of suture thread, airotor was used to remove remaining luting cement. The force, which was required to pull the suture thread from the crown, was equivalent to remove excess cement after setting using hand instrument. Adewumi and Kays ${ }^{11}$ documented SSC aspiration during oral sedation of combination of $10 \mathrm{mg}(0.6 \mathrm{mg} / \mathrm{kg})$ of midazolam with $50 \mathrm{mg}(3 \mathrm{mg} /$ $\mathrm{kg}$ ) of hydroxyzine during the removal of the SSC on 64 prior to the cementation where the crown became dislodged distally and slipped into the airway which was retrieved with bronchoscopy under general anesthesia. Shah and Bhatia ${ }^{2}$ reported an ingestion of posterior esthetic restorative crown where initial crown was prepared for SSC; however, the parents were not satisfied with the esthetics of the crown, therefore they decided to proceed with an esthetic posterior zirconia crown were esthetic restoration and during trial of crown the patient jerked and due to excess salivation the crown slipped out of the hand and the child swallowed the zirconia crown and the crown was found in the patients' stool. Accidents are unforeseen and unpredictable, therefore, always prevention is better than cure holds true in routine clinical procedures. Preventive measures must be practiced religiously by the pediatric dentists in order to avoid accidental swallowing of dental materials.

Initially, the patient exhibited Frankel's behavior rating scale Il; however, after the application of rubber dam, the child became very uncooperative therefore, we decided to proceed the treatment without application of the rubber dam. The method used in our case to prevent accidental crown ingestion has the advantages of simple and easy method, which can be used in routine pediatric dental practice, less time-consuming, which helps in the behavior management. The disadvantages of the procedure are it cannot be applied for a child who exhibits Frankel's behavior rating definitely negative.

\section{Conclusion}

A novel technique of using suture thread and RMGl luting cement is described to prevent aspiration and ingestion of indirect restorations. In case of accidental ingestion, the crown can be easily retrieved immediately by pulling the thread, thereby reducing or even quashes the chance aspiration of zirconia crown. The technique used in our case report can be used as a reserved technique for patients who are unable to accept rubber dam.

\section{References}

1. Oliveira CF, Almeida JF, Troster EJ, et al. Complications of tracheobronchial foreign body aspiration in children: report of 5 cases and review of the literature. Rev Hosp Clin Fac Med Sao Paulo 2002;57(3):108-111. DOI: 10.1590/S0041-87812002000300005.

2. Shah UL, Bhatia R. Ingestion and management of posterior esthetic restorative crown in pediatric dental setup. Int J Prev Clin Dent Res 2018;5:29-33. 
3. Roopa KB, Poornima $P$, Pathak $S$, et al. Foreign body ingestion in a pediatric patient: report of case and review of ingestionlaspiration incident management. Int Dent Med J Adv Res 2015;1(1):1-3. DOI: 10.15713/ins.idmjar.21.

4. Grossman LI. Prevention in endodontic practice. JADA 1971;82(2): 395-396. DOI: 10.14219/jada.archive.1971.0052.

5. Lee JH. Foreign body ingestion in children. Clin Endosc 2018;51(2): 129-136. DOI: 10.5946/ce.2018.039.

6. Kurkciyan I, Frossard M, Kettenbach J, et al. Conservative management of foreign bodies in the gastrointestinal tract. Z Gastroenterol 1996;34:173-177.
7. Katge F, Pammi TH. Tagging of stainless steel crown e an innovative technique!!!. JPFA-India Section 2008;27:106-108.

8. Kher M, Rao A. Lesion management: no removal of carious tissue Kher M, Rao A, ed. Contemporary treatment techniques in pediatric dentistry. Switzerland: Springer; 2019. pp 1-44.

9. An SY, Shim YS. Deciduous teeth using prefabricated zirconia crown in children with early childhood caries. J Dent Hyg Sci 2018;18(3):194200. DOI: $10.17135 /$ jdhs.2018.18.3.194.

10. Blatz MB, Conejo J. Cementation and bonding of zirconia restorations. Compend Contin Educ Dent 2018;39(Suppl 4):9-13.

11. Adewumi A, Kays DW. Stainless steel crown aspiration during sedation in pediatric dentistry. Pediatr Dent 2008;30:59-62. 